developed countries are ageing rapidly, the most dramatic increases are occurring in developing countries. Because most elders prefer to be cared for at home, these profound demographic shifts are driving a global need for home care (HC) at an unprecedented rate. As a result, $\mathrm{HC}$ aide jobs are among the fastest growing occupations and yet their occupational safety and health (OSH) experience is nearly invisible. The Safe Home Care Project at the University of Massachusetts, Lowell USA, funded by the US National Institute for Occupational Safety and Health, was established to protect and promote the OSH of the HC workforce.

Methods We used mixed methods ranging from focus groups to large scale OSH questionnaire surveys, microbiology field studies, and laboratory experimental studies of chemical, biologic, and biomechanical hazards. These studies were performed among HC aides and elders who are $\mathrm{HC}$ recipients.

Results HC aides experience numerous OSH hazards similar to hospitals and nursing homes: back injuries from patient lifting; needlestick injuries; respiratory irritant exposures from cleaning and disinfecting; and serious encounters with violence from patients or family members. Aides also experience hazards not seen in institutional settings: exposure to secondhand smoke, risk of fire from patients smoking cigarettes while on oxygen, patients re-using needles for injections and storing them improperly, and lack of medical equipment for patient lifting. Overall, we found that high quality care delivery depends significantly on HC aide safety.

Discussion $\mathrm{HC}$ aides need $\mathrm{OSH}$ protections. Despite $\mathrm{OSH}$ challenges, the great majority of aides report high job satisfaction due to meaningful relationships with patients and families and to the relative autonomy compared to institutional care work. Interventions should enhance these beneficial aspects of $\mathrm{HC}$ work as well as improve $\mathrm{OSH}$.

\section{SEED SAFETY AND HEALTH WHEN RUSHING TO HELP} ${ }^{1,2,3}$ Michael Riediker, ${ }^{4}$ Magda Stepanyan. ${ }^{1}$ IOM (Institute of Occupational Medicine)
Singapore, Singapore, Singapore; ${ }^{2}$ School of Materials Science and Engineering, Nanyang
Technological University, Singapore, Singapore; ${ }^{3}$ Institut universitaire romand de santé au
travail, University of Lausanne, Epalinges, Switzerland; ${ }^{4}$ The Risk Society, Den Hague, The
Netherlands

\subsection{6/oemed-2018-ICOHabstracts.28}

Introduction Helping local populations develop economic independence is an important aspect of international development programming, and can be critical for longer-term resilience building after major natural disasters. When teaching new skills and methods, one needs to make sure that:

1. those teaching the skills stay healthy while on site, and

2. that the new workforce learns about the essential elements of workplace safety and health (WSH) so that they stay healthy while becoming economically more stable.

Methods The principle to keep both, trainers and trainees safe and healthy during a training and later on is universal. However, many of these interventions happen in ill-controlled situations with many other concerns such as security and administrative challenges related to the exceptional situation. Furthermore, many well-intending teams perceive the situations as 'emergency'. Combined with the hesitance to be 'better treated' than the locals, this can lead to them accepting and taking more risks than they would accept at home.
Result Trainers need to plan how to recognise and teach good safety and health practices in situations where they don't have access to sophisticated or expensive measures. They can set an example to the local population by using WSH methods that are adapted to the specific risks of the solutions. They need to be simple and cheap so that they can later be applied by the locals. Trainers need to emphasise the importance of WSH to prevent the creation of long-term health problems.

Discussion Once development partners and emergency responders understand these WSH-challenges, they can plan for them. It is important to train all stakeholders in how to include WSH aspects. Also donors need to understand the importance of WSH so that they can accept, if not even demand that WSH is given importance also from a perspective of value for money.

\section{MY TRUTH IS BETTER THAN YOURS - HOW TO FIGHT BACK IN THE AGE OF ALTERNATIVE FACTS}

Jani Ruotsalainen. Cochrane Work Review Group, Finnish Institute of Occupational Health in Kuopio, Finland

\subsection{6/oemed-2018-ICOHabstracts.29}

Apparently we live in the time of post-truth and alternative facts. However, we do not have to take this lying down. What we, the scientific community, can offer is a viable alternative, meaning real facts. There are two things that are critical in this: what to do when faced with outright lies or opinions parading as facts and what to offer in their place. The key to both issues is bias. Alternative facts consciously ignore biases and their effects. Somehow a personal gut feeling just trumps scientific reasoning. The best thing we can do is to accept the existence of biases and to try and minimise their effects in what we do. One way to do this is by means of systematic review. It is a means of abstracting a higher level of truth from multiple scientific studies that each examine a similar issue. Whilst synthesising their results and formulating overall conclusions, one explicitly displays the biases affecting both the existing research (what others have done) and the process of synthesis (what you do to combine the results of the studies in one conclusion).

For example, a Cochrane review found high quality evidence that the use of blunt needles appreciably reduces the risk of exposure to blood and bodily fluids for surgeons and their assistants over a range of operations. High quality evidence means that the finding is not significantly affected by biases in the evidence or in the process to combine their results. Future research is unlikely to change this conclusion. Conversely another Cochrane review found very low quality evidence that bullying behaviours may be prevented in the workplace. Future research is very likely to change this conclusion. Know thyself and be open about your failings. That is a sound basis for true facts.

\section{THE ROLE OF EPIDEMIOLOGICAL RESEARCH IN THE PREVENTION OF OCCUPATIONAL ILL HEALTH}

Lesley Rushton. Imperial College London, London, UK

10.1136/oemed-2018-ICOHabstracts.30

Introduction Concerns about occupationally-related diseases that are rare in the general population have provided an 